\title{
Characteristics of Wall Thickness Increase in Pipe Reduction Process using Planetary Rolls
}

\author{
Yuji Kotani, Shunsuke Kanai and Hisaki Watari
}

\begin{abstract}
In recent years, global warming has become a worldwide problem. The reduction of carbon dioxide emissions is a top priority for many companies in the manufacturing industry. In the automobile industry as well, the reduction of carbon dioxide emissions is one of the most important issues. Technology to reduce the weight of automotive parts improves the fuel economy of automobiles, and is an important technology for reducing carbon dioxide. Also, even if this weight reduction technology is applied to electric automobiles rather than gasoline automobiles, reducing energy consumption remains an important issue. Plastic processing of hollow pipes is one important technology for realizing the weight reduction of automotive parts. Ohashi et al. [1],[2] present an example of research on pipe formation in which a process was carried out to enlarge a pipe diameter using a lost core, achieving the suppression of wall thickness reduction and greater pipe expansion than hydroforming.

In this study, we investigated a method to increase the wall thickness of a pipe through pipe compression using planetary rolls. The establishment of a technology whereby the wall thickness of a pipe can be controlled without buckling the pipe is an important technology for the weight reduction of products. Using the finite element analysis method, we predicted that it would be possible to increase the compression of an aluminum pipe with a $3 \mathrm{~mm}$ wall thickness by approximately $20 \%$, and wall thickness by approximately $20 \%$ by pressing the hollow pipe with planetary rolls.
\end{abstract}

Keywords —Pipe-Forming, Wall Thickness, Finite-element-method

\section{INTRODUCTION}

$\mathrm{I}_{\mathrm{i}}^{\mathrm{N}}$ $\mathrm{N}$ recent years, thickness addition by press forming, which increases the wall thickness of parts requiring strength, has received attention in the field of press forming as one processing method for promoting weight reduction in automotive parts. Thickness addition by press forming is a processing method that controls wall thickness of a press formed product by adjusting the geometric shape of the die, thus causing the flow of material from a single piece of sheet material during forming to increase the wall thickness in parts where strength is required. However, as the processing time of press forming is extremely short, the contact portion between the die and the sheet material can vary with time, making the flow of the sheet material extremely complex. Also, it is difficult to visualize the phenomena within the die during forming, which further complicates research of the sheet material flow. As a result, it is extremely difficult to design a die that could control the flow of sheet material so as to thicken a specific part. Thus, in the manufacturing process, a prototype

Yuji Kotani, Shunsuke Kanai and Hisaki Watari are with the Department of Production Science and Technology, Graduate school of Engineering, Gunma University, 29-1, Hon-cho, Ota, Gunma 373-0057 Japan (phone: +81 27650 2250; fax: +81 27650 2235; e-mail: t09802207@gunma-u.ac.jp ). die is produced first, followed by checking the shape of the processed material obtained thereby, and then repeatedly modifying the design and altering the die in order to define the shape of the die. As a result, cost and price increases pose a problem in die design. Also, structural components such as frames are often produced by subjecting hollow pipes to plastic deformation in order to reduce weight. In research on processing cylindrical pipes into shapes such as rectangular tubes, the method of extroll forming has been widely examined [3]. However, although extroll forming allows the forming of long, uniformly shaped objects, it is difficult to process objects in which the shape changes part way. Hydroforming is frequently used for manufacturing when a pipe with a complex shape is to undergo plastic processing [4]. Hydroforming is a processing method for forming a shape by employing the pressure of a liquid to expand the object to a target shape. Although a certain amount of axial force is applied to prevent the occurrence of tears, wall thickness is fundamentally reduced during processing in this method. Processing using a lost core has been proposed for processing by internal pressure in order to minimize the reduction in wall thickness and prevent large deformation[1],[2], but this only minimizes the reduction in wall thickness, and processing whereby the wall thickness of the pipe is increased essentially does not occur. As a method of compressing a pipe to an arbitrary diameter, Kiuchi et al. propose a planetary roller reducer [5]. It is thought that a planetary roller reducer, which uses planetary rolls, could also be used to control pipe wall thickness in combination with a die that squeezes the pipe.

In this study, we examine the partial increase in wall thickness through pipe processing using planetary rolls, and we investigate the effects of forming conditions on variations in wall thickness on the processed material by evaluating the flow of material during forming and the thickness on each part of the formed product, for the purpose of deforming the pipe without buckling it due to pipe compression using planetary rolls. We then make proposals concerning elements such as the flow of materials and increasing wall thickness in pipe processing using planetary rolls.

\section{INCREASES IN PIPE WALL THICKNESS DUE TO PIPE COMPRESSION}

Examining Compression Methods - Feed-Through Pipe Compression : It is thought that pipe wall thickness can be increased during processing through deforming to shorten the length of the pipe by applying axial force to the pipe. If this method is used on a long pipe, buckling will occur before the wall thickness increases. It is conceivable that wall thickness 
could be increased by reducing the radius of the pipe in order to prevent buckling. A method could be envisaged that reduces pipe diameter and increases wall thickness by passing the pipe through a die with a conical shaped hole as shown in Figure 1. In this case, because the pipe diameter would be reduced as it passes through the conical shaped die, a load in the pipe axial direction would be generated in addition to that in the pipe radial direction, as shown in Fig. 2. Because of this load in the axial direction, if the pipe diameter becomes too small, buckling of the pipe will occur similar to the method of increasing wall thickness by shortening the pipe length

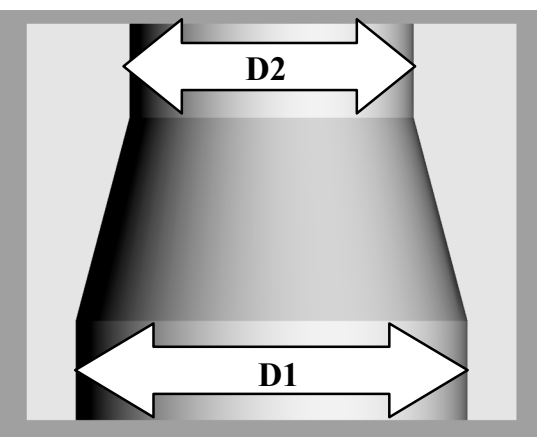

Fig. 1 Example cross section of the compression die

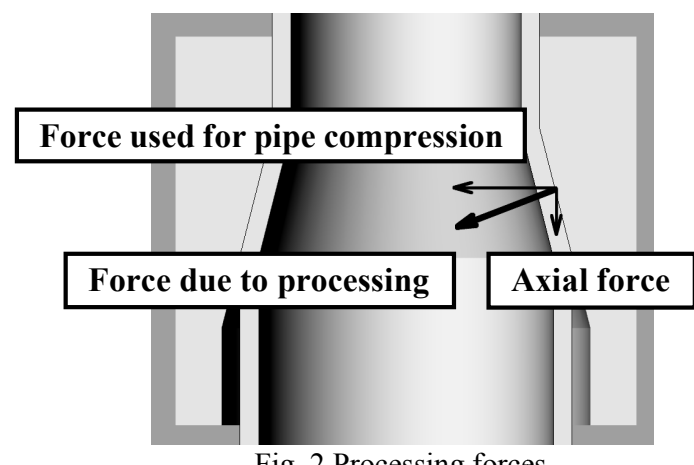

Fig. 2 Processing forces

It was reported that compression up to a diameter of $26 \mathrm{~mm}$ is possible in a pipe with a diameter of $30 \mathrm{~mm}$, a wall thickness of $2 \mathrm{~mm}$ and a length of $100 \mathrm{~mm}$ [6]. According to similar analysis, with a pipe with the same diameter of $30 \mathrm{~mm}$ but with a length of $200 \mathrm{~mm}$ and a thickness of $3 \mathrm{~mm}$, if the bottom edge of the pipe is fixed in the die, buckling occurs at compression to a diameter of $28 \mathrm{~mm}$, as shown in Fig. 3. Also, Fig. 4 shows the diameter ratios before and after compression and the results of compression. From this it is clear that compression by passing a pipe through a conical shaped opening is very difficult when the pipe is long.

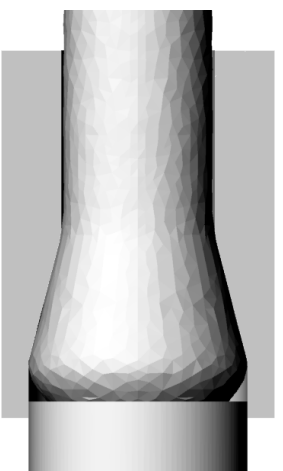

Fig.3 Pipe Buckling

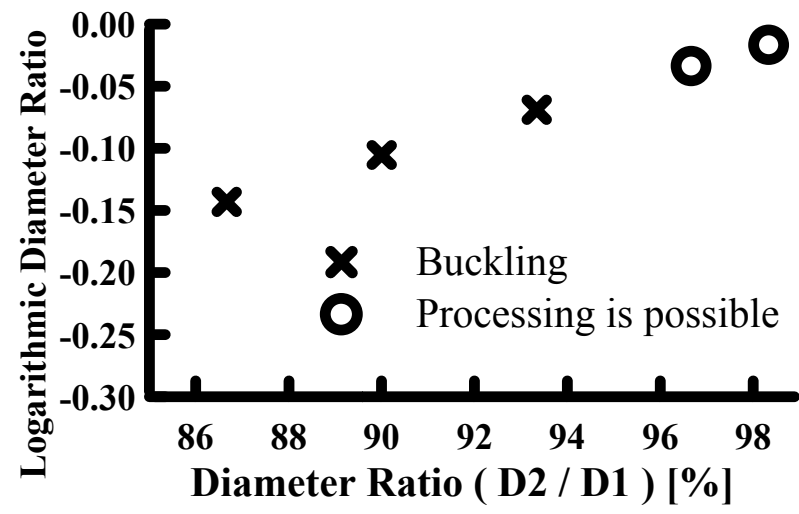

Fig. 4 Diameter Ratios Before and After Compression and Compression Results

Pipe Compression Using Planetary Rolls : As stated above, a method of reducing pipe radius and increasing wall thickness by passing a pipe through a conical shaped opening places a large load in the axial direction of the pipe, causing the pipe to buckle, thus making it impossible to reduce the diameter all at once. For this reason, we investigated a method of compression by rotating rolls having multiple slopes and passing the pipe through these rolls, as shown in Fig. 5. Carrying out compression by rotating rolls around the pipe reduces the load in the axial direction, making it possible to achieve a comparatively large compression.

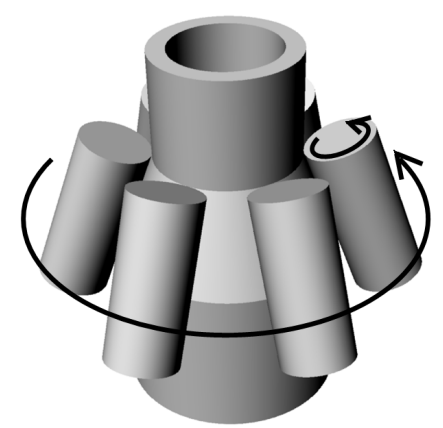

Fig. 5 Compression by Planetary Rolls 


\section{COMPRESSING USING Planetary Rolls}

Experiment Conditions and Results : To make the apparatus as simple as possible, rather than rotating the planetary roll around the pipe, a method was selected in which a milling machine as shown in Fig. 6 was used, wherein the pipe was rotated as it was fed through. The experiment conditions are shown in Table 1. Also, roller angles $(\alpha)$ and ( () , as shown in Fig. 7, are respectively the angle in which the roll is directed towards compressing the pipe, and the angle of the direction orthogonal to the roll compression against the pipe's central axis. By enlarging ( $(3)$, axial force can be generated in accordance to the roll's rotation during compression processing. The experiment was carried out using six rollers and an angle (ß) of 0 .

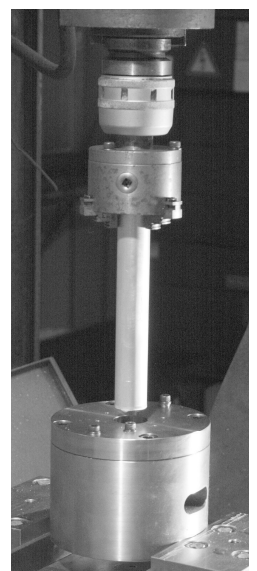

(a)Set for Work

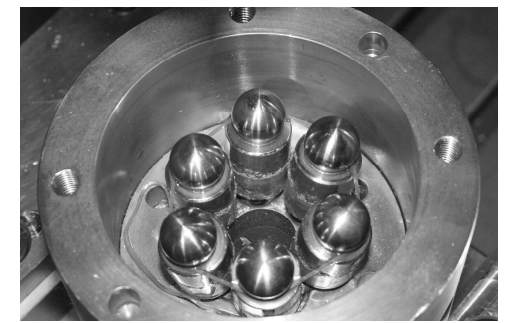

(b)Interior of Experiment Apparatus

Fig. 6 A Look at the Experiment

TABLE I

EXPERIMENT CONDITIONS

\begin{tabular}{|c|c|}
\hline Pipe Diameter [mm] & 30.0 \\
\hline Pipe Thickness $[\mathrm{mm}]$ & 2.0 \\
\hline Pipe Length $[\mathrm{mm}]$ & 200.0 \\
\hline Material & Aluminum (A1070T) \\
\hline Roller Angle $(\alpha)$ [degrees] & 0 \\
\hline Roller Angle $(ß)[$ degrees] & 15 \\
\hline Roller Revolutions $[\mathrm{rpm}]$ & 5 \\
\hline Pipe Feeding Speed $[\mathrm{mm} / \mathrm{min}]$ & \\
\hline
\end{tabular}

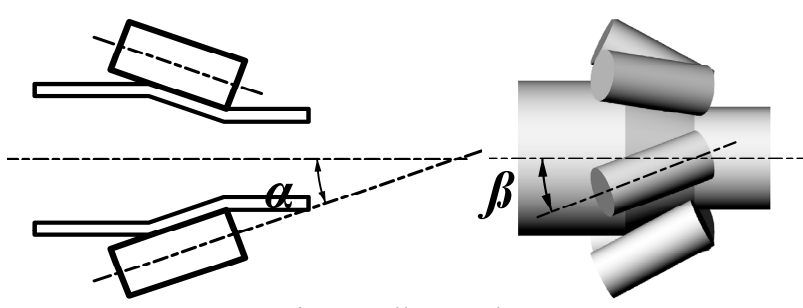

Fig. 7 Roller Angles

The compression results of the pipe after the experiment was carried out are shown in Fig. 8. Fig. 8 makes it clear that compression can be carried out without buckling the pipe. Strain in each direction of the pipe before and after compression processing is shown in Fig. 9.

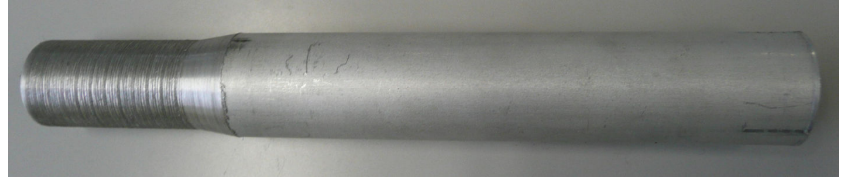

Fig. 8 An Example of a Processed Pipe

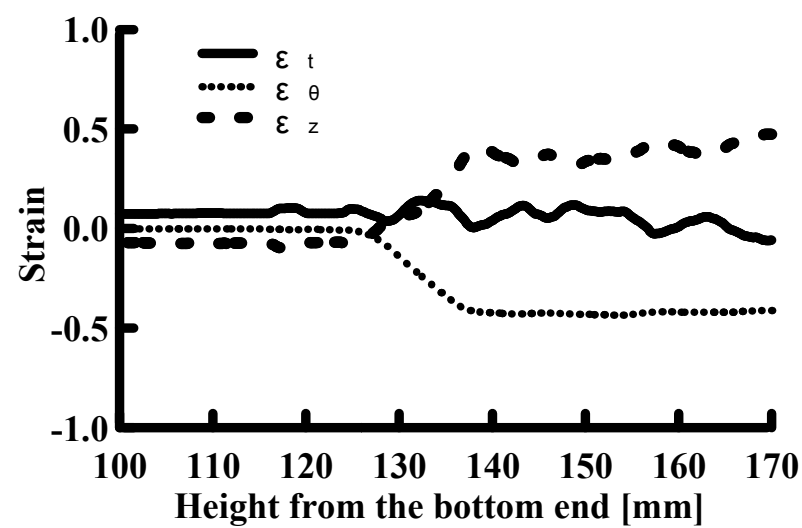

Fig. 9 Strain in Each Direction

In this experiment, because a milling machine was used as the experimental apparatus, the speed at which the pipe was fed was limited by the load capacity limit on the milling machine's central axis. Although there was no buckling, tensile strain was thus generated in the axial direction as shown in Fig. 9, and although there was about a 5\% increase in wall thickness in the area of the compression processing, a large increase did not occur.

Analysis Conditions and Results : First, analysis was carried with the same conditions as the experiment above, and after comparing changes in pipe thickness analysis was performed regarding the occurrence of pipe buckling and increases in wall thickness under conditions that applied relatively large axial force on the pipe. Analysis conditions are shown in Table 2. In regard to roller angle $(\beta)$, because the roller rotates during formation processing, the side on which the axial force works to feed the processing material of the pipe into the die is considered positive, and the side on which force works in the direction for pulling the pipe during processing is negative. Of the analyses given in Table 2 , the analysis with conditions of a roller revolution of $15[\mathrm{rpm}]$ and a pipe feeding speed of 5 $[\mathrm{mm} / \mathrm{min}]$ corresponds to the experiment described above. The positional relationship of the roll and pipe at the time of analysis is shown in Fig. 10.

An example of the pipe shape after compression according to the analysis is shown in Fig. 11. Also, Fig. 12 is a diagram showing a cross-section of the analysis results carried out under conditions virtually identical to the experiment, and also shows a comparison of ratios of sheet thickness in the area of compression processing in the experiment and the analysis. In Fig. 12, the horizontal axis is set to zero at the point where processing ends. The range from roughly -12 to 0 is the range during processing by the roll. 
TABLE II

ANALYSIS CONDITIONS

\begin{tabular}{|c|c|c|}
\hline Diameter [mm] & & 30.0 \\
\hline Pipe Length [mm] & & 200.0 \\
\hline Material & \multicolumn{2}{|c|}{ Aluminum (AL-1100) } \\
\hline Thickness [mm] & 2.0 & 3.0 \\
\hline Roller Revolution [rpm] & 15 & 2 \\
\hline $\begin{array}{l}\text { Pipe Feeding Speed } \\
{[\mathrm{mm} / \mathrm{min}]}\end{array}$ & 5,50 & 60 \\
\hline Roller Angle $(\alpha)$ [degrees] & 10 & 10 \\
\hline Roller Angle (ß) [degrees] & 0 & $-30,-20,-10,0,10,20,30$ \\
\hline $\begin{array}{c}\text { Diameter After } \\
\text { Compression } \\
{[\mathrm{mm}]}\end{array}$ & 26 & 26,24 \\
\hline
\end{tabular}
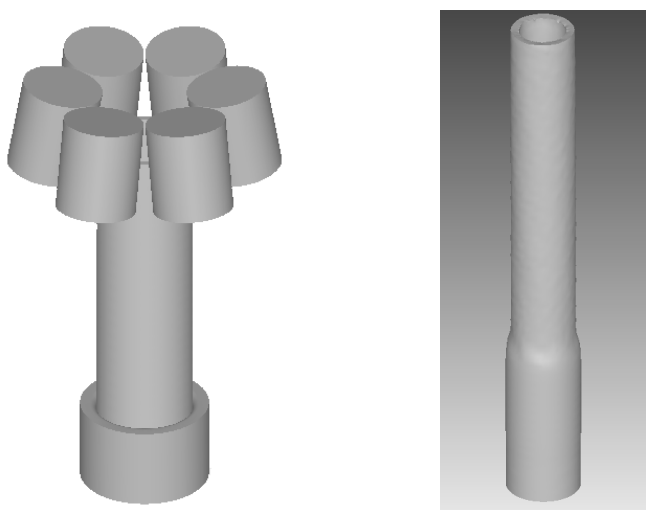

Fig.11 An Example of an Analysis Result

Fig. 10 Analysis of Compression by Rolls

In Fig. 12, it is apparent there is a slight increase in thickness in the experiment due to axial load before processing, and it is apparent there is a phenomenon in which the state of thickness increase during processing differs slightly from the experiment due to the relationship of the analysis mesh, but a similar trend is exhibited in the post-processing shape between the analysis and the experiment, and it is clear that recreating the experiment through analysis is possible.

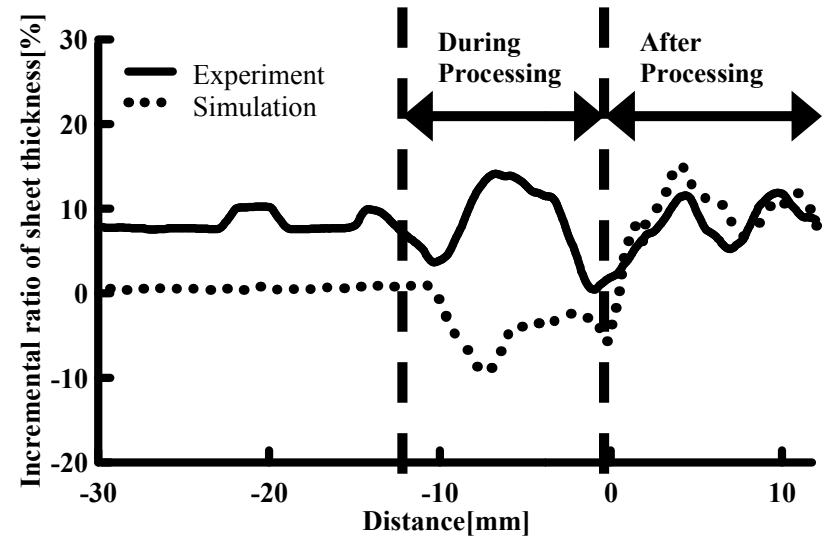

Fig.12 Ratio of Sheet Thickness in Experiment and Analysis Under the Same Conditions

Fig. 13 shows the ratio of sheet thickness of a pipe in a case in which the roller revolution is 15 [rpm], the pipe feeding speed is $50[\mathrm{~mm} / \mathrm{min}]$, which equates to raising the pipe feeding speed 10 times. Fig. 13 makes it clear that when the speed of the pipe is raised, the thickness of the pipe increases approximately $20 \%$.

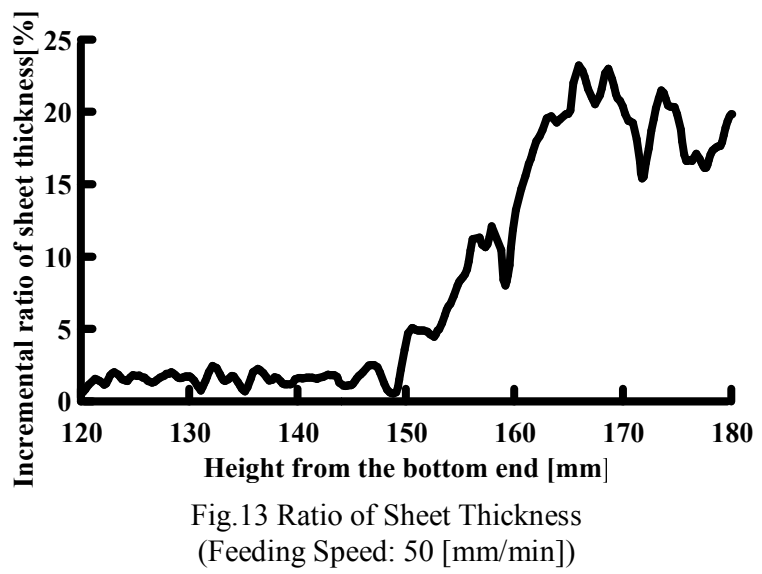

Supposing that the pipe will be fed through the rotating roller at high speed using press processing or the like, the roller angle (B) was varied and analysis was carried out in which the pipe feeding speed was raised in contrast to the roller revolutions, such as using a roller revolution of 2 [rpm] and a pipe feeding speed of $60[\mathrm{~mm} / \mathrm{min}]$. The resulting quality of the compression condition under each condition in the analysis is shown in Table 3. An example of a shape that would be considered a "bad" result of the analysis is shown in Fig. 14. Also, Fig. 15 shows the ratio of sheet thickness when the roller angle ( $(B)$ is set to $30^{\circ}$ and the diameter is compressed to $24 \mathrm{~mm}$, which produced the best shape and thickness increase in this analysis.

TABLE III

QUALITY OF THE COMPRESSION CONDITION

\begin{tabular}{|c|c|c|c|}
\hline \multicolumn{2}{|c|}{} & \multicolumn{2}{|c|}{ Diameter after Compression [mm] } \\
\cline { 3 - 4 } \multicolumn{1}{c|}{} & -30 & Good & Good \\
\cline { 2 - 4 } & -20 & Buckling & Bad \\
\cline { 2 - 4 } Roller & -10 & Buckling & Bad \\
\cline { 2 - 4 } Angle (B) & 0 & Buckling & Bad \\
\cline { 2 - 4 }$[$ degree $]$ & 10 & Buckling & Bad \\
\cline { 2 - 4 } & 20 & Buckling & Good \\
\cline { 2 - 4 } & 30 & Bad & Good \\
\hline
\end{tabular}

From the analysis results, it can be seen that, depending on conditions, a phenomenon can occur in which the pipe will be processed into a polygonal shape under the condition that the pipe feeding speed is made large in relation to the rotational speed of the planetary rolls surrounding the pipe. The number of the angles of the polygonal shape produced is one less than the number of planetary rolls. It is thought that this is due to processing occurring as the center of the pipe rotates slightly around the rotational center of the rolls like a gear inside the rolls during processing. Also, it can be seen that the post-processing shape is better when the rolls are turned towards the central axis of the pipe. 


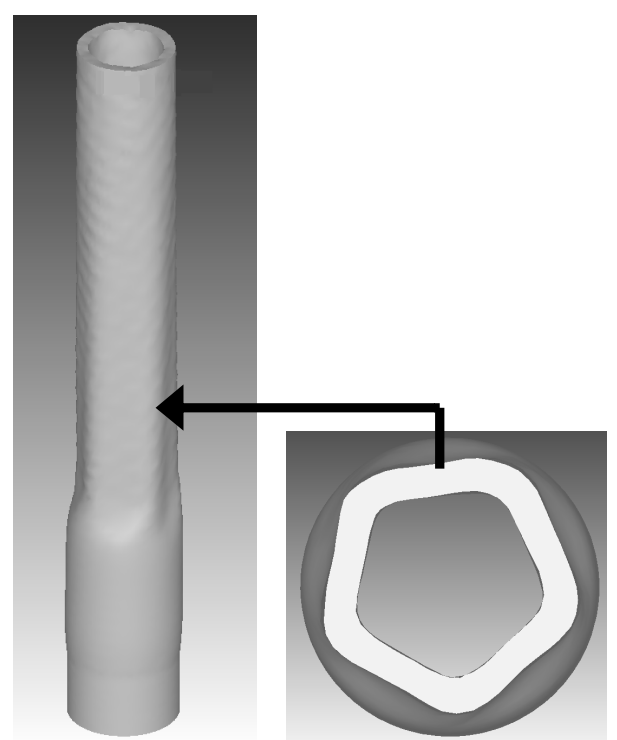

Fig. 14 A Bad Shape of Compression

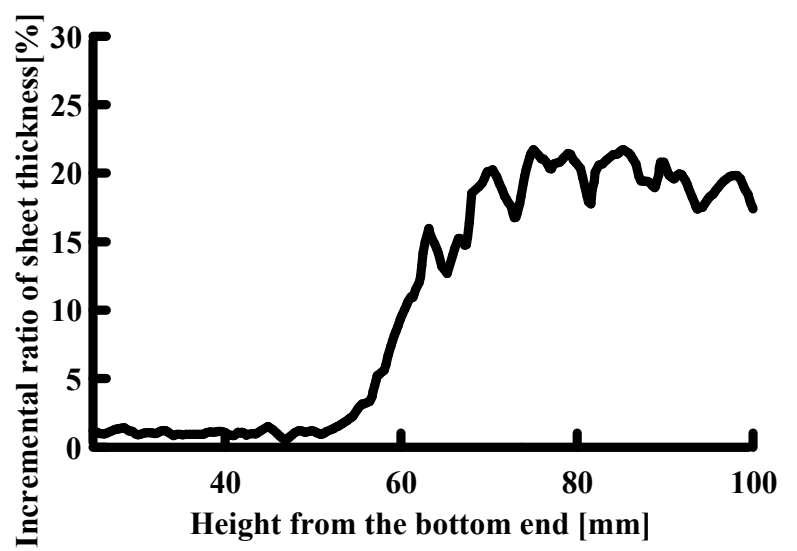

Fig. 15 Thickness Increase Rate (Diameter:24 mm, (ß): $30^{\circ}$ )

\section{CONCLUSION}

In this study, we examined a method for increasing pipe wall thickness by pipe compression using planetary rolls. Through the experiment, we confirmed that it is possible to compress a pipe without buckling it by processing using planetary rolls, and through analysis we estimated that it is possible to increase wall thickness and compression by approximately $20 \%$ respectively in an aluminum pipe by changing the pipe feeding speed and the angle of the rolls.

\section{REFERENCES}

[1] T.Ohashi, K.Hayashi, Journal of Materials Processing Technology Vol.138 (2003) 560-563.

[2] T. Ohashi, K.Matsui, Y.Saotome, Journal of Materials Processing Technology, Vol.113 (2001) 98-102.

[3] Takuo Nagamachi, Onoda Yoshitomi, Kimura Sadao, Kitawaki Takeo, Materials Transactions, Vol.45, No.4 (2004) 1328-1334.

[4] A. Shirayori, S. Fuchizawa, H. Ishigure, M. Narazaki, Journal of Materials Processing Technology Vol.139 (2003) 58-63.

[5] Manabu KIUCHI, Ken SHINTANI, Joaunal of JSTP Vol.38 no.433(1997-2) 177-182.

[6] Yuji Kotani, Hisaki Watari and Akihiko Watanabe, Advanced Materials Research Vol. 320 (2011) 456-461. 